\title{
Current Clinical Trials and Vaccine Development Strategies for Corona Virus Disease (COVID-19)
}

\author{
Abhi Bhadra (i), Shweta Singh (D), Shaswat Chandrakar (D), Vanshika Kumar (D), \\ Sakshi Sankhla (D), Sayuj Raj T. (D) and E. Selvarajan*
}

Department of Genetic Engineering, School of Bioengineering, SRM Institute of Science and Technology, Kattankulathur - 603 203, Tamil Nadu, India.

\section{Abstract}

Severe acute respiratory syndrome coronavirus 2 (SARS-CoV-2) has reached global epidemic status claiming more than $319 \mathrm{~K}$ lives and affecting more than $4.81 \mathrm{M}$ people and counting worldwide. Considering the severity of the situation and low recovery rate many research institutions and pharmaceutical industries are rushing to learn more about this new virus and the morbid physiology of this disease with effective diagnostic methods, therapeutic agents and vaccines. Various approaches are highlighted for comparing the possible treatment methods available for COVID-19 some of which are BCG vaccination on COVID-19 and Non-pharmaceutical interventions, drug based clinical trials of Hydroxychloroquine-Azithromycin, chloroquine, lopinavir/ritonavir, ChAdOx1 nCoV-19, Remdesivir, Stem Cell therapy and mesenchymal stromal cell therapy, etc.

Keywords: Lopinavir, Ritonavir, Chloroquine, Hydroxychloroquine, Stem Cell Therapy, Cytokines, Remdesivir

*Correspondence: selrajan@gmail.com; +91 9843430425

(Received: May 08, 2020; accepted: May 21, 2020)

Citation: Bhadra A, Singh S, Chandrakar S, et al. Current Clinical Trials and Vaccine Development Strategies for Corona Virus Disease (COVID-19). J Pure Appl Microbiol. 2020;14(suppl 1):979-988. doi: 10.22207/JPAM.14.SPL1.36

C The Author(s) 2020. Open Access. This article is distributed under the terms of the Creative Commons Attribution 4.0 International License which permits unrestricted use, sharing, distribution, and reproduction in any medium, provided you give appropriate credit to the original author(s) and the source, provide a link to the Creative Commons license, and indicate if changes were made. 


\section{INTRODUCTION}

Coronaviruses are a large group of viruses mainly divided into six genera. These viruses affect the body through symptoms ranging from common cold to severe acute respiratory syndrome (SARS) or Middle East respiratory syndrome (MERS) ${ }^{1}$. Novel coronavirus was reported in December 2019 in Wuhan city, Hubei province, China ${ }^{2}$. This virus was reported to be proginated from a new strain previously unidentified in humans ${ }^{3}$. The International Committee on Taxonomy of Viruses named 2019-nCoV as SARS-CoV- $2^{4}$ and the disease inflicted by it as coronavirus disease or COVID-19. Coronavirus belongs to the family Coronaviridae that possess single-strand, positive-sense RNA genome ranging from $26-32 \mathrm{kbp}$ in size. The Coronaviridae family ${ }^{5}$ contains six genera sheltering Alpha-coronavirus (alphaCoV), Beta-coronavirus (betaCoV), Delta-coronavirus (deltaCoV) ${ }^{6}$, Gammacoronavirus (gammaCoV), Bifinivirus, Torovirus ${ }^{7}$. Coronaviruses are named after their surface morphology (viewed microscopically) representing glycoprotein spikes like a crown or "corona".

There has been a lot of research going on to find the cure, some candidates have come up with a cure. Although, they are still in the early stages of trials some of them have actually shown quite good results. This article describes the comparative results of possible treatment methods of BCG vaccines, lopinavir/ ritonavir, hydroxychloroquine-azithromycin, chloroquine, remdesivir, chadox 1 based vaccines, and mesenchymal stem cell therapy options.

\section{BCG Vaccination}

Covid-19 virus has infected over 300,000 individuals and led to over 13,000 deaths in its initial three months; nonetheless the pattern of development isn't uniform8. Mechanistic proof exists to counsel that vaccination with Bacillus Calmette-Guérin (BCG), will have protecting effects against virus infection. BCG vaccines are accessible since the first twentieth century, and are necessary in many countries similar to Japan and South Korea and plenty of a lot of for cover against TB (TB) infection'. BCG vaccines aren't mandated in many different countries, together with Italian Republic, the US, and different developed countries. BCG vaccines contain a live attenuated strain of eubacterium bovis, the inducive agent of TB. The BCG vaccination has been steered to induce heterologous immunity against non-mycobacterial pathogens. The immunogen is assumed to engineer metabolic and epigenetic alterations, leading to the promotion and production of proinflammatory cytokines following exposure to a stimulation. Preliminary reports from medicine studies counsel that counties with universal BCG vaccination programs are wedged less by Covid-19, leading researchers to hypothesize that BCG vaccination might supply some level of heterologous immunity against COVID-19 ${ }^{10}$.

\section{METHODS}

Human services laborers are at the bleeding edge of the coronavirus infection (COVID-19) pandemic. Members will be human services laborers in Australian emergency clinic locales. They will be randomized to get a solitary portion of BCG antibody, or no BCG immunization. Members will be lined up for a year with customary cell phone instant messages (up to week after week) and studies to recognize and detail COVID-19 disease. Extra data on extreme malady will be gotten from clinic clinical records and government databases. Blood tests will be gathered before randomisation and at a year to decide presentation to serious intense respiratory disorder coronavirus 2 (SARS-CoV-2). Where required, swab/blood tests will be taken at sickness scenes to survey SARSCoV-2 disease ${ }^{11}$.

\section{Lopinavir/Ritonavir}

A number of clinical trials have performed to find a cure for the treatment of COVID-19 one of which is the lopinavir/ritonavir based clinical tria $\left.\right|^{12}$. Lopinavir being the human immunodeficiency virus (HIV)-1 protease inhibitor is administered in the patients in fixed-dose combination with ritonavir, a potent CYP3A4 inhibitor that boosts the concentration of lopinavir. This combination of drugs blocks the main protease of SARS-Cov-1 inhibiting viral replication ${ }^{13}$.

The outcomes of the first 18 patients who were affected by SARS-CoV-2 in Singapore were reported by Young et al. Out of these 18, 5 were given the LPV/r treatment. It was a 14-day course with guidelines at a dose of $400 \mathrm{mg} / 100 \mathrm{mg} \mathrm{(2}$ capsules/tablets) by mouth twice a day. A ratio of 1:1 of lopinavir and ritonavir were used either with standard care or without it. All the confirmed cases of adults were given this treatment. A lot of 
other patients were also given the same dosage for the authenticity of this method of treatment. Cao et al reported the results of an open-label randomized trial comparing LPV/r 400/100 mg twice daily to standard care for the treatment of COVID-19 pneumonia. clinical improvement of the patients was set as the primary endpoint defined from randomization time to either improvement upto two points on the seven-category ordinal scale or discharge from treatment depending on the scenario ${ }^{14}$.

Hydroxychloroquine-Azithromycin, chloroquine

The clinical treatment options based on known compounds whose physiological efficacy are validated across wide spectrum of diseases are taken into consideration simultaneously with the discoveries of new treatment methods ${ }^{15}$. One such drug is chloroquine that is an antimalarial drug has shown its effectiveness against a broad range of viruses and since it's an old drug, its sideeffects as well as interactions with other drugs (if administered together) are already known along with its possible dosage for treatment of COVID-19 associated pneumonia ${ }^{16}$. hydroxychloroquine, a chloroquine-analogue, is perceived as a better candidate for these trials because of its safety profile attributing towards less hindrance with other drugs and higher daily dosage ${ }^{17}$.

Before the trials were conducted to determine the viral clearance properties of these drugs (on COVID-19/SARS-CoV-2) the consents were taken from the patients (or their kin) by giving full disclosure about the effects of these drugs after administration ${ }^{18}$. The proper Dosages of chloroquine as well as hydroxychloroquine were administered in various clinical trials along

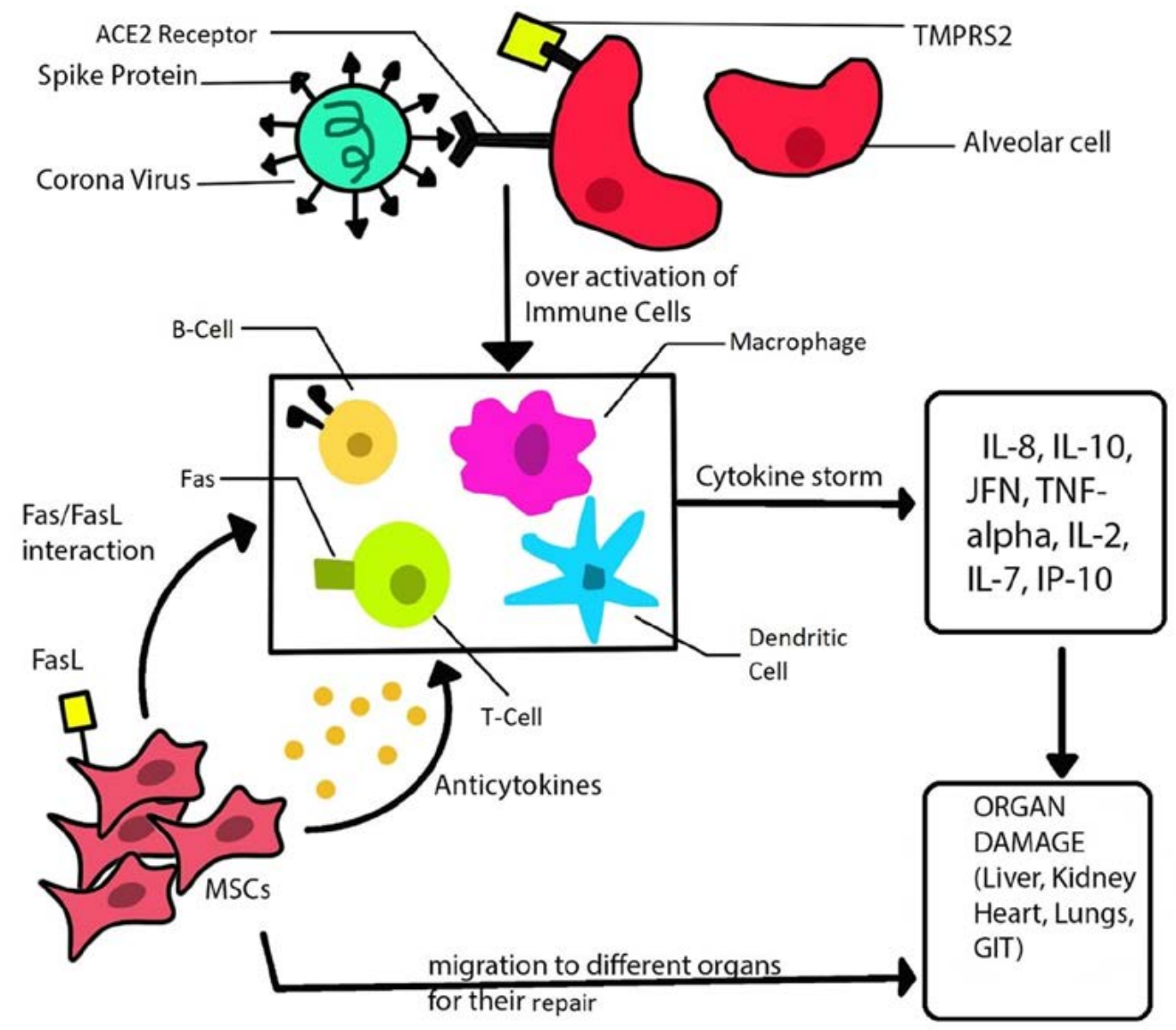

Fig. 1. MSCs (extracted from human umbilical cord blood) transferred intravenously triggering immune response which activate the immune cells. Thus MSCs releases EVs and Anti-cytokines which migrate to damaged organs and also overcome the hyper-activation of immune cells and cytokines. 
with complementary compounds as chloroquinephosphates or hydroxychloroquine-azithromycin mixture in order to enhance the clinical outcome and viral clearance properties of these drugs ${ }^{19}$. All the trials were population based and were recorded with patient distributions in broadly three categories: i) Patients suffering from upper respiratory tract infection, ii) Patients suffering from lower respiratory tract infection and iii) Negative controls which consisted of patients refusing the trial of these drugs. The patient demographic under these trials mainly focused on adults and elderly since children were recorded to show mild symptoms of COVID-19/SARS-CoV-2 induced pneumonia. The participants of these trials were administered with regular dosages of the drugs for a period of 6-10 days (varying according to trials). Nasopharyngeal swabs ${ }^{21}$ were taken from the patients every day for the duration of the trials at regular intervals to check viral proliferation and clinical effect of the drug on symptoms. The known effects of chloroquine as an antiviral is that it alkalises phagolysosomes which influences the low $\mathrm{pH}$ reliance of viral replication, including fusion and coat uncovering. Similarly, hydroxychloroquine hinders the surface level $\mathrm{pH}$ of the cell membrane and inhibit the fusion of the virus to the cell membrane. It can also inhibit nucleic acid replication, glycosylation of viral proteins, virus assembly, new virus particle transport. Azithromycin on the other hand was administered alongside Hydroxychloroquine to prevent severe respiratory tract infections. Hydroxychloroquine is studied extensively with respect to higher dosage concentrations because of its safety profile for toxicity ${ }^{20}$.

Mesenchymal stem cell therapy

Multiple treatments have been studied for treating corona virus. Most of which consist of drug therapies. But treatments are supportive, not curative for the disease ${ }^{21}$.

When virus enters in our body, our immune system produces a responsive counter attack of massive production of inflammatory cytokines. And this may cause organ damage leads to death of patients. So if cytokine storm is prevented from its formation then it's a key factor to treat patients.

Finally, in China Umbilical Mesenchymal Stem cell were studied for their potential role against Corona virus because of limited availability of effective modalities. As a result of this treatment, the in-patient/out-patient numbers decreased in the ICUs ${ }^{22}$.

Stem cell's contribution

The cure for the virus is dependent on patients' own immunity. MSC appeared to generate natural immunity against the corona virus by secreting Anti-inflammatory factors. These factors suppress the cytokines and inhibit the over activated immune system.

They also promote endogenous repair by modifying their microenvironment. When MSCs are intravenously injected, they are said to gather in the lungs, then potentially improve the pulmonary components and improve lung functioning.

MSCs have the differentiative abilities and immunomodulation that helps them interact with their paracrine secretions with B cells $T$ cells and dendritic cells.

Case presentation 1

(According to Liang and colleagues)

MSC isolated from human umbilical cord potentially improved the immune response as well as repaired affected tissues in a 65-year-old female affected from COVID-19 critically. This clinical trial had excellent safety. Clinicians injected the MSCs intravenously thrice $\left(5 \times 10^{\wedge} 7\right.$ cells each time) every third day. The second dosage period marked the improvement time slot where the trachea cannula was managed and the serum bilirubin, $\mathrm{C}$-reactive protein (CRP), alanine transaminase/ aspartate transaminase (ALT/AST) reduced.

\section{Case presentation 2}

This trial was certified on $5 / 2 / 2020$ by Beijing 302 Hospital and was the first phase of clinical trials to observe the reliability of UCMSCs therapy safely for the patients infected by Covid-19. In this 20 patients were volunteered in which 10 patients were received 3 intravenous injection of Stem cell with conventional therapy. And 10 patients were given conventional therapy, used as control. The clinical symptoms like pulmonary imaging, side effects, and 28-days mortality, immunological characteristics (immune cells, inflammatory factors, and microenvironment modulation) will be checked for 180 days. Due to immunoregulatory function of MSCs, patients recover from the disease and those who are used 
Table 1. Parameters of clinical symptoms before and after MSC Therapy

\begin{tabular}{lll}
\hline Clinical signs and symptoms & Before MSCs Therapy & After few days of Therapy \\
\hline Fever $\left({ }^{\circ} \mathrm{C}\right)$ & $38.5-38.8$ & Normal \\
Shortness of breath & Present & normal breathing \\
cough & present & No cough \\
sputum & present & No sputum in throat \\
Oxygen saturation & Low like $89 \%$ to $91 \%$ & Rose upto $95 \%-97 \%$ \\
Respiratory rate & $27-33$ & $21-22$ \\
C-reactive protein $(\mathrm{ng} / \mathrm{ml})$ & $105-191$ & Normal level \\
Absolute count of lymphocyte $\left(\times 10^{\wedge} 9 / \mathrm{L}\right)$ & $0.23-0.35$ & Normal level \\
White cell count $\left(\times 10^{\wedge} 9 / \mathrm{L}\right)$ & $11-12.56$ & Normal range \\
Absolute count of Neutrophils $\left(\times 10^{\wedge} 9 / \mathrm{L}\right)$ & $9-11.33$ & Normal range \\
\hline
\end{tabular}

as control had severe pulmonary defects and were taken to $\mathrm{ICU}^{23}$.

\section{Case presentation 3}

In an ongoing preliminary, the specialist takes 7 patients ( 1 basically genuine, 4 genuine and 2 normal) contaminated with the coronavirus and were given one portion of undifferentiated organism treatment. What's more, they were contrasted and 3 patients in the benchmark group ( 3 genuine) who didn't get immature microorganism.

Crown infection explicitly perceives the angiotensin I changing over chemical 2 receptor (ACE2) by its spike protein. the ACE2 receptor is extensively present on the human cells surface, particularly the alveolar sort II cells (AT2) and narrow endothelium. Likewise, cell serine protease TMPRSS2 for HCoV-19 Spike protein preparing is significant in the host cell section and spread. Be that as it may, the bone marrow, lymph hubs, thymus, and the spleen, invulnerable cells like
$T$ and $B$ lymphocytes, and macrophages are negative for ACE2. So this is the motivation to take mesenchymal undifferentiated cell as potential treatment. To treat understanding. The immunoregulatory activity of MSCs are likewise expanded by the actuation of TLR receptor present on MSCs, which is invigorated by pathogen-related particles, for example, LPS or twofold abandoned RNA from infection like the HCoV- $19^{24}$.

The treatment was followed for 14 days. All the 7 patients with immature microorganism treatment recouped. be that as it may, in the benchmark group, one patient passed on, another patient created ARDS. Just a single patient in the benchmark group was steady. No further multifaceted nature was found in the treatment gathering. In the treated gathering of patients following a couple of long stretches of treatment the oxygen immersion, biomarkers for irritation and tissue injury like CRP, aspartic aminotransferase, creatine kinase movement and

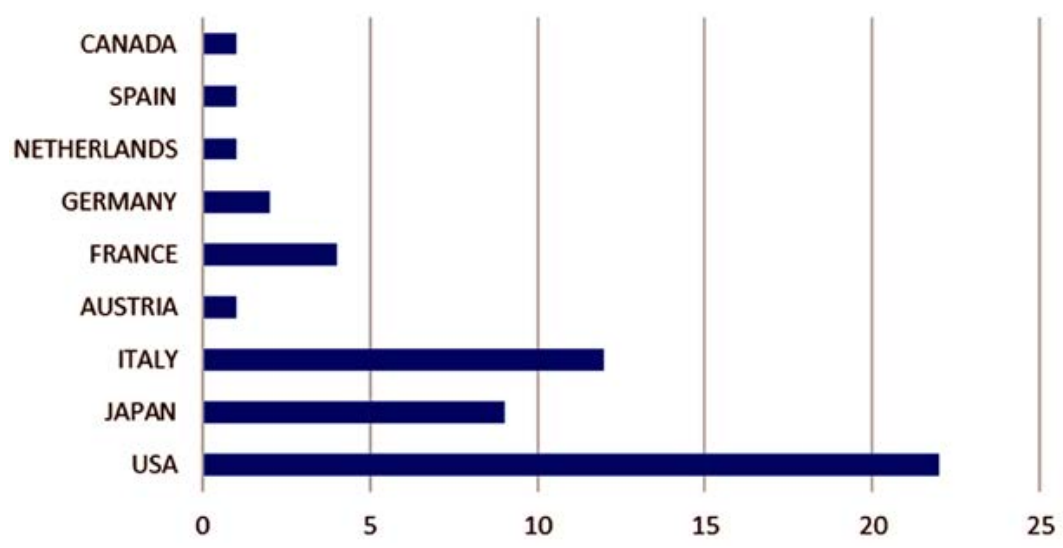

Fig. 2. Nationality of patients 
Table 2. Account of patients and their nationality

\begin{tabular}{ll}
\hline Nationality & No of People \\
\hline USA & 22 \\
Japan & 9 \\
Italy & 12 \\
Austria & 1 \\
France & 4 \\
Germany & 2 \\
Netherlands & 1 \\
Spain & 1 \\
Canada & 1
\end{tabular}

myoglobin were standardized. Through the CT output of the lungs, enhancements were found in the lungs. Impediments incorporate little example size and present moment follow up.

Remdesivir

Remdesivir (RDV, GS-5734) is a monophosphoramidate pro-drug diastereomeric of the nucleoside analogue of adenine GS-441524. Inside of cells, GS-441524 is converted into the form pharmacologically active triphosphate (GS-443902), which in turn is able to inhibit RNAdependent virus polymerase ${ }^{25}$. RDV has shown broad spectrum antiviral activity with good efficacy in-vitro and in vivo on animal models against various RNA viruses, genetically unrelated but similar to SARS-CoV-2, such as SARS-CoV and coronavirus from Middle Eastern respiratory syndrome (MERS-CoV). For EBOLA they have shown promising results in inhibiting MERS-CoV, SARS-CoV, bat CoV which are different strains of the SARS-CoV 2 (COVID-19) ${ }^{26}$.

Procedure

Remdesivir (GS-5734) is a monophosphate drug and a nucleoside analogue. RDV was developed as a drug against EBOLA Virus which showed didn't show much promising results back then. Later it proved to be useful in inhibiting the two strains of coronaviruses causing the SARS-CoV and the MERS-CoV which led to the thought of using Remdesivir as a potential inhibitor of SARSCov 2. Even though there was no previous studies or surety that the RDV drug was going to be useful against 2019-nCoV.Many other compounds were also notably useful against the 2019-nCoV.The factor that would make Remdesivir stand out had to be found out by the researchers ${ }^{27}$.

The program was conducted from the 25th of January to 7th of March $2020^{30}$. The chosen patients were given a starting dose of Remdesivir of $200 \mathrm{mg}$ on day 1 followed by $100 \mathrm{mg}$ for the rest of 9 days. The program was open and accepted any number of patients. A total of 61 patients were accepted for his program after checking their status and demographics of the 61 patients accepted for the program four patients (8\%) discontinued Remdesivir treatment prematurely: one because of worsening of pre-existing renal failure ${ }^{31}$, one because of multiple organ failure, and two because of elevated aminotransferases, including one patient with a maculopapular rash ${ }^{28}$.

The gender ratio was 1:4 and the age range of the selected patients varied from 23 years to 82 years and the median range was 64 years. And in another experiment in china,

The patients were divided into different groups for this experiment. All patients belonged to three Chinese nationality. Informed consent was obtained from eligible patients or their substitute decision-makers (for minors or people with decision lacking capacity) by study physicians or other trial staff with delegated responsibilit ${ }^{29}$.

Group A was the experimental group with $200 \mathrm{mg}$ of RDV on day 1 and $100 \mathrm{mg}$ of RDV for the rest 9 days.

Group B had placebo in $350 \mathrm{ml}$ saline solution for day 1 and $250 \mathrm{ml}$ for the rest of the days.

ChadOX1

Recombinant adenoviruses are among the most encouraging methods for antibody antigen conveyance framework. As of late, the advancement of new vectors has concentrated on serotypes to which the human populace is less presented so as to go around prior enemy of vector resistance.

ChAdOx1 nCoV-19 is genetically modified adenovirus vector synthesized from a virus "ChAdOx1" ${ }^{30}$, which is a debilitated form of a typical cold infection (adenovirus) that causes diseases in chimpanzees, that has been hereditarily changed. In this way, that it is inconceivable for it to cultivate inside human body. Due to this is it is one of potential option for vaccine for Covid- 19. Gene is added to the ChAdOx1's genetic makeup using recombinant DNA technology that is used to synthesize proteins called Spike glycoprotein. This protein is generally found on the periphery of 
SARS-CoV-2 and assumes a fundamental job in the disease pathway of the SARS-CoV-2 infection. The SARS-CoV-2 coronavirus utilizes its spike protein to tie to ACE2 receptors on human cells to pick up section to the cells and cause an infection.

Although, it's still in the early phases of clinical based trial but according to the oxford university press the scientist are hoping to strengthen once immunity of population. Currently this adenoviral vector is still in the first phase of clinical trial. Nearly 1200 people have come and have participated for the trial which started in April, $2020^{31}$.

The primary focal point of these preliminary is to see whether this antibody is going to neutralize COVID-19, in the event that it won't cause any unfavourable reactions and in the event that it actuates great invulnerable reactions. The portion utilized in this preliminary was picked dependent on past encounters with other ChAdOx1 based vaccines.

The members won't know whether they have gotten the ChAdOx1 nCoV-19 immunization until the finish of the preliminary.

The initial days of immunizations was arranged as follows:

After getting vaccination the participant is given an e- dairy to record the symptom or any changes for 7 days. And if the participant gets any unwanted side effects then medical supervision is provided by the professionals ${ }^{32}$.

Day 1

The initial two members will be inoculated, one with the ChAdOx1 nCoV-19 antibody and one with the control immunization. Members checked for 48 hours.

Day 3

A further six members will be inoculated, three with the ChAdOx1 nCoV-19 antibody and three with the control immunization. Members checked for 48 hours.

Day 5

Progress to inoculating more members.

\section{RESULTS AND DISCUSSION}

There are many institutions working to developed potential cure for the disease. These incorporate immunizations, meds like Remdesivir and hydroxychloroquine along with a mix of medications treatment.
In any case, medications are steady, not therapeutic for the infection. If there should arise an occurrence of BCG vaccination 178 nations had information from every one of the 3 sources and molded the reason of our examination. Current national projects of BCG immunization exist in 131 nations; 21 nations don't have any present program of national BCG inoculation; and for 26 nations standing is obscure. Over going before 15 days, frequency of Covid-19 was 38.4 per million in nations with BCG inoculation contrasted with 358.4 per million inside the non-participation of such a program. The passing rate was $4.28 /$ million in nations with BCG programs contrasted with 40/ million in nations without such a program. Nations with national program of entire populace BCG immunization appear to claim a lower mortality rate from Covid-19. This may result to the betterrealized clinical claim of BCG inoculation. Inside the absence of a specific immunization against Covid-19, populace-based BCG inoculation could have an undertaking in diminishing the effect of this unwellness and is being concentrated in an exceedingly imminent preliminary.

Similarly, in lopinavir/ritonavir based clinical trial outcomes in Singapore trial: After the treatment initialized, 3 patients showed less oxygen requirement while the other 2 had a respiratory failure. Two of 5 patients (40\%) shoed clearance of viral shedding on treatment although 4 of 5 patients faced adverse events. On a bright side 6 total patients from Korea and China showed a positive response with decreased viral activity and clinically improved state. Outcomes reported by Chau: No significant difference was seen in time to clinical improvement or mortality rate between the LPV/r group and the standard care group.

While on the other hand According to Gautret et al. it was reported that there was complete viral clearance on the nasopharyngeal swabs after $5^{\text {th }}$ and $6^{\text {th }}$ day of combination drugs of hydroxychloroquine and azithromycin. Hydroxychloroquine alone worked at more than half the efficacy (57.1\%). Although Hydroxychloroquine performed better in-vitro anti SARS CoV 2 activity than chloroquine. Still a lot more sample study are to be done in order to provide any substantiated information.

Talking about vaccination the chadOX1 is In the initial trial out of 1200 participants the 
Immunizations produced using the ChAdOx1 infection have been given to in excess of 320 individuals to date and have been demonstrated to be sheltered and all around endured, despite the fact that there can be transitory reactions, for example, a temperature, cerebral pain or sore arm which are quit accepted . any further development has not been made public by the institution as the initial trial is still running.

While research with Mesenchymal stem cell therapy. The analysis through10x scRNAseq exposes that MSCs are ACE2- and TMPRSS and secrete anti-inflammatories to forestall the cytokine storm and have characteristic invulnerability to HCov-19. Results from mass cytometry, the infection disease caused all outcapacity breakdown of the lymphocytes and of the entire invulnerable framework. MSCs incites develop dendritic cells into a functioning Jagged-2subordinate administrative dendritic cell populace. These communications with various dendritic cells cause the immune system from Th1 toward Th2 responses.

Due to viral infection causes inflammation and C-reactive protein act as biomarker for and at the same time the host produces cytokines specifically TNF $\alpha$, IL-6, MCP1 and IL- 8 by T cells. And over activation of immune system. When MSCs injected intravenously, some part of it accumulate in the lungs and improve alveolar epithelial cells, fibrosis and lungs function. So MSC therapy inhibits the over activation of the immune system and promote endogenous repair by improving the microenvironment.

Due to its action of improving microenvironment and immunosuppression ability, the levels of pro-inflammatory cytokines and chemokines were decreased at long last in serum. Also, it draws in less no. of mononuclear/ macrophages to the lung. Signs are sent to initiate increasingly administrative dendric cells to the inflamed tissue. Additionally, the lungs improvement is finished by increased IL-10 and VEGF. At last, the patients with serious COVID19 pneumonia endure the most noticeably terrible condition and entered recuperation.

\section{CONCLUSION}

BCG vaccine requires more human trials to learn more about its antiviral proficiency against SARS-CoV-2. Many countries still need to inculcate BCG vaccination in their programs to provide substantial data against this virus.

Lopinavir/Ritonavir combination shows promise against immunodeficiency complications. However, the output is low with viral clearance decreasing their efficacy.

Hydroxychloroquine-azithromycin combination shows more promise for viral clearance in general. With properties inhibiting nucleic acid replication, glycosylation of viral proteins, virus assembly and new virus particle transport this drug combination becomes a potential treatment option.

Treatment using mesenchymal stem cell can provide long term curative outcomes of this disease but is still a long way from becoming an immediate source of treatment for COVID-19 which still requires more evidential positive results from its administration.

Remedisivir has shown good antiviral efficacy against RNA viruses genetically unrelated but similar to SARS-CoV-2 and coronavirus from MERS-CoV in animal models. Its results against COVID-19 has more potential than other drugs but lacks human test results.

ChAdOx1 based vaccines are cultivated with adenovirus vectors which express spike protein similar to corona virus thus showing promise as another vaccine development strategy against COVID-19 but the lack of human trials are still prominent in this endeavour.

\section{ACKNOWLEDGMENTS}

None.

\section{CONFLICT OF INTEREST}

The listed author(s) declare no conflict of interest in any capacity, including competing or financial.

\section{AUTHORS' CONTRIBUTION}

All authors listed have made a substantial, direct and intellectual contribution to the work, and approved it for publication. 
FUNDING

None.

\section{ETHICS STATEMENT}

This article does not contain any studies with human participants or animals performed by any of the authors.

\section{DATA AVAILABILITY}

All datasets generated or analyzed during this study are included in the manuscript and/or the Supplementary Files.

\section{REFERENCES}

1. Culp W C. Coronavirus Disease 2019. A A Pract. 2020;14(6):e01218-e01218. https://doi.org/10.1213/ XAA.0000000000001218

2. Franks TJ \& Galvin JR. Coronavirus. in Viruses and the Lung: Infections and Non-Infectious ViralLinked Lung Disorders. 2014;109-116. https://doi. org/10.1007/978-3-642-40605-8_13

3. Cui J, Li F, Shi ZL. Origin and evolution of pathogenic coronaviruses. Nature Reviews Microbiology. 2019;17(3):181-192. https://doi.org/10.1038/s41579018-0118-9

4. Zhu $\mathrm{N}$ et al. A novel coronavirus from patients with pneumonia in China, 2019. N. Engl. J. Med. 2020;382:727-733. https://doi.org/10.1056/ NEJMoa2001017

5. Liu C et al. Research and Development on Therapeutic Agents and Vaccines for COVID-19 and Related Human Coronavirus Diseases. ACS Cent. Sci. 2020;6(3):315331.6. https://doi.org/10.1021/acscentsci.0c00272

6. Kooraki S, Hosseiny M, Myers L, Gholamrezanezhad A. Coronavirus (COVID-19) Outbreak: What the Department of Radiology Should Know. J. Am. Coll. Radiol. 2020;17(4):447-451. https://doi.org/10.1016/j. jacr.2020.02.008

7. Chan JFW et al. A familial cluster of pneumonia associated with the 2019 novel coronavirus indicating person-to-person transmission: a study of a family cluster. Lancet. 2020;395:514-23. https://doi. org/10.1016/S0140-6736(20)30154-9

8. Weiss SR, Navas-Martin S. Coronavirus Pathogenesis and the Emerging Pathogen Severe Acute Respiratory Syndrome Coronavirus. Microbiol. Mol. Biol. Rev. 2005;9(4):635-64. https://doi.org/10.1128/ MMBR.69.4.635-664.2005

9. Gursel M, Gursel I. WITHDRAWN: Is global BCG vaccination coverage relevant to the progression of SARS-CoV-2 pandemic? Med. Hypotheses. 2020; 109707. https://doi.org/10.1016/j.mehy.2020.109707

10. Szigeti R, Kellermayer D, Kellermayer R. BCG protects against COVID-19? A word of caution. medRxiv. 2020; 20056903. https://doi. org/10.1101/2020.04.09.20056903

11. Curtis N. BCG Vaccination to Protect Healthcare Workers Against COVID-19 (BRACE). Retrieved from http//clinicaltrials.gov/ct2 (Identifier NCT04327206), 2020.

12. Yao TT, Qian JD, Zhu WY, Wang Y, Wang G Q. A systematic review of lopinavir therapy for SARS coronavirus and MERS coronavirus-A possible reference for coronavirus disease-19 treatment option. Journal of Medical Virology. 2020;92:556-563. https:// doi.org/10.1002/jmv.25729

13. Chu CM et al. Role of lopinavir/ritonavir in the treatment of SARS: Initial virological and clinical findings. Thorax. 2004;59(3):252-256. https://doi. org/10.1136/thorax.2003.012658

14. Cao B et al. A Trial of Lopinavir-Ritonavir in Adults Hospitalized with Severe Covid-19. N. Engl. J. Med. 2020;382(19):1787-1799. https://doi.org/10.1056/ NEJMoa2001282

15. Yao $X$ et al. In Vitro Antiviral Activity and Projection of Optimized Dosing Design of Hydroxychloroquine for the Treatment of Severe Acute Respiratory Syndrome Coronavirus 2 (SARS-CoV-2). Clin. Infect. Dis. 2020; pii: ciaa237. https://doi.org/10.1093/cid/ciaa237

16. Yazdany J, Kim AHJ. Use of Hydroxychloroquine and Chloroquine During the COVID-19 Pandemic: What Every Clinician Should Know. Ann. Intern. Med. 2020;m20-1334. https://doi.org/10.7326/M20-1334

17. Devaux CA, Rolain JM, Colson P, Raoult D. New insights on the antiviral effects of chloroquine against coronavirus: what to expect for COVID-19? Int. J. Antimicrob. Agents. 2020;12:105938. https://doi. org/10.1016/j.ijantimicag.2020.105938

18. Guastalegname M, Vallone A. Could chloroquine / hydroxychloroquine be harmful in Coronavirus Disease 2019 (COVID-19) treatment? Clin. Infect. Dis. 2020;pii: ciaa321. https://doi.org/10.1093/cid/ciaa321

19. Gautret $P$ et al. Hydroxychloroquine and azithromycin as a treatment of COVID-19: results of an open-label non-randomized clinical trial. Int. J. Antimicrob. Agents,. 2020;20:105949. https://doi.org/10.1016/j. ijantimicag.2020.105949

20. Gao J, Tian Z, Yang X. Breakthrough: Chloroquine phosphate has shown apparent efficacy in treatment of COVID-19 associated pneumonia in clinical studies. BioScience Trends. 2020;14(1):72-73. https://doi. org/10.5582/bst.2020.01047

21. Bari E, et al. Mesenchymal Stromal Cell Secretome for Severe COVID-19 Infections: Premises for the Therapeutic Use. Cells. 2020;9(4). pii: E924. https:// doi.org/10.3390/cells9040924

22. Atluri S, Manchikanti L, Hirsch JA. Expanded Umbilical Cord Mesenchymal Stem Cells (UC-MSCs) as a Therapeutic Strategy in Managing Critically III COVID-19 Patients: The Case for Compassionate Use. Pain Physician. 2020;23(2):E71-E83

23. Shetty AK. Mesenchymal stem cell infusion shows promise for combating coronavirus (COVID-19)-induced pneumonia. Aging and Disease. 2020;11(2):462-464. https://doi.org/10.14336/AD.2020.0301

24. Leng $Z$ et al. Transplantation of ACE2- Mesenchymal stem cells improves the outcome of patients with covid-19 pneumonia. Aging Dis. 2020;11(2):216-228. https://doi.org/10.14336/AD.2020.0228

25. Wang $\mathrm{M}$ et al. Remdesivir and chloroquine effectively 
inhibit the recently emerged novel coronavirus (2019nCoV) in vitro. Cell Research. 2020;30(3):269-271. https://doi.org/10.1038/s41422-020-0282-0

26. Gordon CJ, Tchesnokov EP, Feng JY, Porter DP, Gotte M. The antiviral compound remdesivir potently inhibits RNAdependent RNA polymerase from Middle East respiratory syndrome coronavirus. Journal of Biological Chemistry. 2020;295(15):4773-4779. https://doi. org/10.1074/jbc.AC120.013056

27. Gordon $\mathrm{CJ}$ et al. Remdesivir is a direct-acting antiviral that inhibits RNA-dependent RNA polymerase from severe acute respiratory syndrome coronavirus 2 with high potency. J. Biol. Chem. 2020; RA120.013679. https://doi.org/10.1074/jbc.RA120.013679

28. Yethindra V. Role of GS-5734 (Remdesivir) in inhibiting SARS-CoV and MERS-CoV: The expected role of GS5734 (remdesivir) in COVID-19 (2019-nCoV)-VYTR hypothesis. Int. J. Res. Pharm. Sci. 2020;1-6. https:// doi.org/10.26452/ijrps.v11iSPL1.1973
29. Agostini ML et al. Coronavirus susceptibility to the antiviral remdesivir (GS-5734) is mediated by the viral polymerase and the proofreading exoribonuclease. MBio. 2018;9(2):e00221-18. https://doi.org/10.1128/ mBio.00221-18

30. Alharbi NK et al. ChAdOx1 and MVA based vaccine candidates against MERS-CoV elicit neutralising antibodies and cellular immune responses in mice. Vaccine. 2017;35(30):3780-3788. https://doi. org/10.1016/j.vaccine.2017.05.032

31. Abd El-Aziz TM, Stockand JD. Recent progress and challenges in drug development against COVID-19 coronavirus (SARS-CoV-2) - an update on the status. Infection, Genetics and Evolution. 2020;83:104327. https://doi.org/10.1016/j.meegid.2020.104327

32. Li $Z$ et al. Development and clinical application of a rapid IgM-IgG combined antibody test for SARS-CoV-2 infection diagnosis. J. Med. Virol. 2020;1-7. https:// doi.org/10.1002/jmv.25727 